\title{
HMS: A MATLAB simulator of the Harris model of associative learning
}

\author{
Holger SCHULTHeIS \\ Universität Bremen, Bremen, Germany
}

AND

AnNa ThORWart and Harald Lachnit

Philipps-Universität Marburg, Marburg, Germany

\begin{abstract}
Harris (2006) recently proposed a new elemental model of the processes involved in associative learning. Although Harris explicated all relevant mathematical and conceptual details of the model in his article, implementing a computer simulation of his model requires considerable programming expertise and work. We therefore present the Harris model simulator (HMS), a MATLAB simulator of Harris's model. HMS provides a graphical user interface for manipulating all essential parameter values and for controlling the simulation process, the graphical visualization of the simulation course, and the numerical results. HMS is available free of charge from www.staff.uni-marburg.de/ lachnit/harris/. HMS allows researchers to easily derive and evaluate predictions for the Harris model, and it will therefore facilitate insights into the mechanisms of associative learning.
\end{abstract}

Animals as well as humans often face situations in everyday life in which several individual stimuli are present at the same time, all of which might be related to a specific effect or outcome. Hence, a basic question that theorists have grappled with for decades is whether learning attaches independently to each element that constitutes the sensory array or instead to the array as a whole. Theories in which the former view is adopted, so-called elemental theories, assume that responding to an array composed of many elements is a direct function of the values attached to the elements themselves, with the whole array having no separate value over and above that of its constituent parts. In contrast, theories in which entire arrays are assumed to be the basic units of learning (configural theories) assume that responding is driven by knowledge about the whole array, independently of its individual parts.

Traditionally, many well-known effects in the field of associative learning have been successfully explained in an elemental manner, with the assumptions that stimulus components are represented as separate entities and that the overall associative strength of a compound is based on the algebraic sum of the associative strengths of its components. This elemental summation principle is incorporated into many theories of associative learning (e.g., Mackintosh, 1975; Pearce \& Hall, 1980; Rescorla \& Wagner, 1972; Wagner, 1981). This simple idea - as exhibited by the Rescorla-Wagner model-is, however, not able to solve a number of discrimination learning problems. In a negative-patterning discrimination, for example, the conditioned stimuli (CSs) A and B are followed by an uncon- ditioned stimulus (US) when they are presented alone, but not when they are presented together $(\mathrm{A}+, \mathrm{B}+, \mathrm{AB}-)$. $\mathrm{A}$ modified version of the Rescorla-Wagner model (Wagner $\&$ Rescorla, 1972) is able to deal with the fact that different species are able to solve this discrimination; it does so by proposing the formation of a unique cue as an additional element when $\mathrm{A}$ and $\mathrm{B}$ are presented together. This unique cue allows different responding to $\mathrm{A}$ and $\mathrm{B}$ when they occur together than when they are presented alone, because the unique cue becomes associated with the absence of food, and thus is able to counteract the prediction of food on the basis of A and B.

Configural approaches, on the other hand, treat stimulus patterns as distinct configurations, so that associations operate on the configuration as a whole. In contrast to all elemental approaches, Pearce $(1987,1994,2002)$ assumes that stimuli are always processed configurally. In his conception, the elements of a compound collectively enter into one, single association, and each stimulus and each compound take on independent associative strengths. In this way, the configural model can straightforwardly account for the acquisition of discrimination problems like negative patterning: $\mathrm{A}+$ and $\mathrm{B}+$ trials each result in the development of an excitatory association between the configural unit A and the US and between the configural unit $B$ and the US, respectively, whereas $A B$ - trials result in the development of an inhibitory association between a configural unit $\mathrm{AB}$ and the US.

In summary, configural theories account for the phenomena of learning in terms of configural representations, 
whereas elemental theories do so in terms of elemental representations. Although there has been a rich and productive history of research on the elemental/configural (or part/whole) distinction, this issue is not settled yet. On the one hand, there are results that support and contradict each view. On the other hand, the necessity of configural representations per se has been fundamentally questioned. For example, Wagner and Brandon (2001) showed that Pearce's configural theory is isomorphic with the elemental inhibited-elements model, which later was expanded into the so-called replaced-elements model (Wagner, 2003; Wagner \& Brandon, 2001; see also Schultheis, Thorwart, \& Lachnit, 2008).

Recently, another elemental alternative to configural accounts of associative learning was introduced by Harris (2006). His model is similar to a modified stimulussampling theory (Estes, 1973, 1994), in which each stimulus representation consists of a sampling of elements, and the elements of different stimuli influence each other's activation. For example, elements that are shared by stimuli are more strongly activated when these stimuli are presented in compound. All of the elements compete for entry into a limited-capacity attention buffer on the basis of changes in their activation weights. Elements with a large increase in activation displace weakly activated elements from the buffer and thus receive an activation boost (i.e., are made more salient). Consequently, the elements inside the buffer are always the most active ones. The capacity of the buffer is defined in terms of activation. At any point in time, the sum of the activations of the elements inside the buffer must not exceed a certain value (i.e., the buffer capacity). As a result, both the number of elements in the buffer and the buffer threshold (i.e., the amount of increase in activation a single element needs to exceed in order to enter the buffer) vary according to the average activation weight of the elements in the buffer.

The attention buffer increases and prolongs the activation of the elements it contains. Elements within the buffer form excitatory associations with one another and inhibitory associations with the weaker elements outside the buffer. The change of associative strength of a CS element during conditioning depends on its activation weight, making the activation weight functionally equivalent to the salience parameters employed in the other models mentioned above. Excitatory conditioning occurs between activated CS elements and US elements that are activated into the attention buffer. Inhibitory conditioning or extinction occurs when US elements are activated outside the buffer. Preexisting links connecting elements (both across and within stimuli) change their strength during conditioning. The present model assumes partial connectivity between elements - that is, that each element is connected to a subset of the total number of elements. According to Harris (2006), this is comparable to assuming complete interconnectivity, but with variability among those connections in terms of their effectiveness at supporting associations (e.g., variations in the "beta" parameter).

Harris's (2006) conception has several advantages in comparison with previously existing approaches. For instance, it is able to predict the detrimental influence of redundant cues on a feature-negative discrimination (see also the examples provided online with the software). Although the model also does have difficulties with some phenomena (e.g., retrospective revaluation), recent evidence suggests that overall, Harris's conception might be a powerful theory of associative learning (see Lachnit, Schultheis, König, Üngör, \& Melchers, in press; Shanks, Lachnit, \& Melchers, 2008). Applying the model, however, requires considerable programming expertise and work, and thus, simulations might not be easily available for researchers working on associative learning theories.

In the present article, we therefore offer a program that can be intuitively operated, called the Harris model simulator (HMS), to simulate the predictions of the associative learning theory suggested by Harris (2006). This software allows for specifying all relevant parameters of the simulation via a graphical user interface (GUI). It then takes care of setting up the simulation and graphical presentation of the learning process and outcome. In the following discussion, we will describe the components and use of HMS in more detail.

\section{HMS}

HMS is written in MATLAB and has been tested with MATLAB 6.5 and higher, on both Windows and Linux operating systems. The software package can be obtained in its most recent version (currently, 1.5) free of charge from www.staff.uni-marburg.de/ lachnit/harris/. To give an overview of the simulator, we will describe its GUI, explicate the structure of the files needed for a simulation, outline computational complexity issues, and finally exemplify the simulator's standard use with one particular discrimination problem. Implementational and mathematical details of the essential parts of HMS are described in the Appendix.

\section{Graphical User Interface}

The GUI of HMS (HMS.m; see Figure 1A) is structured in four different segments. The first segment, located at the top of the GUI, comprises two fields to indicate the locations of the files necessary for the simulation. The second segment is located at the left middle of the GUI and displays the general settings, defined by six parameters. The third segment, positioned at the middle right, displays the advanced settings, defined by four parameters. The last segment, to be found at the bottom of the GUI, comprises three buttons for controlling the simulation. The rest of this section consists of a more detailed description of each of the four segments, in turn.

Both fields in the file segment require a full path to a file. For each field, one can either specify the path by hand or use the "Browse" button on the right to pick the desired file. The first field ("Trial definition file") holds the path to the file containing the definitions for the CSs (compounds) presented throughout the discrimination problem to be simulated. By default, HMS assumes that trial definition files have a ".tr" ending. The second field ("Evaluation definition file") holds the path to the file containing the definitions for the CSs (compounds) for 
A

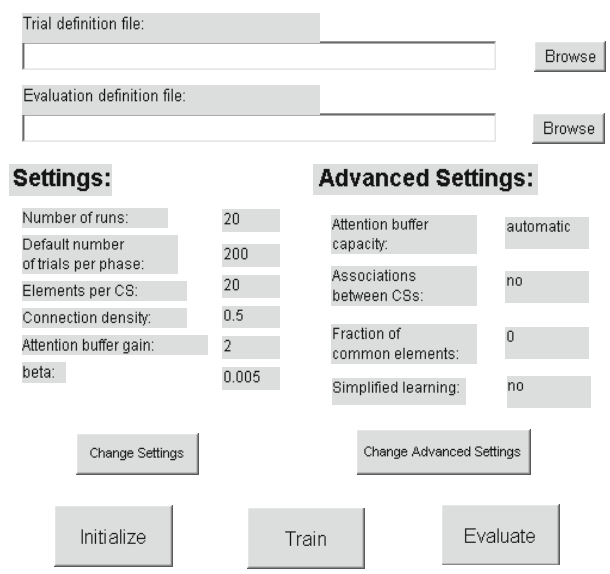

B

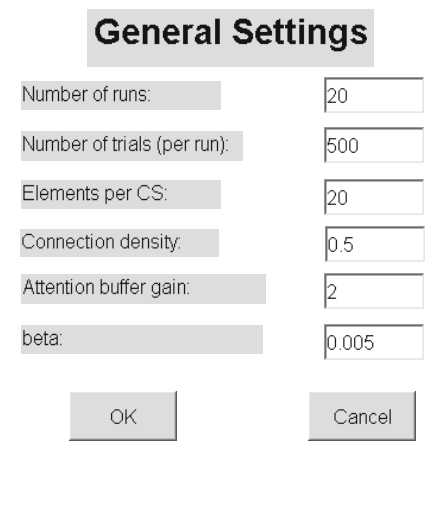

\section{C}

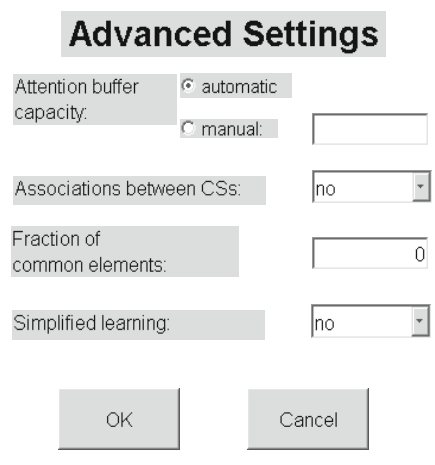

Figure 1. The graphical user interface of HMS. (A) Main window. (B) Dialog box for changing the general settings. (C) Dialog box for changing the advanced settings.

which responding should be evaluated after learning. Evaluation files by default have a ".ev" ending. The precise structure of the different file types will be discussed in detail below.

General settings. The six general settings parameters ${ }^{1}$ can be changed via the General Settings dialog box (see Figure 1B), which opens when the "Change Settings" button on the GUI is pressed. The available parameters, from top to bottom, are described below.

Number of runs. Since the model uses random numbers to determine the intensities of the elements of each stimulus and the existence of connections between elements, different runs of the model may give different results. To give an impression of how the model will behave on average with particular parameters, the model is simulated $x$ times, and the average result of these $x$ runs is taken as the model output. The "Number of runs" parameter indicates $x$, with a default value of 20 .

Default number of trials per phase. In HMS, each run consists of one or more phases, with each phase comprising a set of CSs (or compounds). One simulation trial consists of the presentation of all CSs (or compounds) given for one phase in the trial definition file (see below). There are two ways to specify the number of trials for a phase. On one hand, one can use the "Default number of trials per phase" set in the GUI, and on the other, one can define the number of trials for each phase individually in the trial definition file (see below). The value from the GUI is used for each phase for which the number of trials is not specified in the trial definition file. The preset value for the parameter in the GUI is 200 trials.

Elements per CS. As mentioned above, each CS is represented by a number of elements sampled from a (larger) set of elements. A free parameter of the model"Elements per CS" - determines how many elements are sampled for each CS. This parameter can be specified by using the given field. In line with the values employed by Harris (2006), the default value of this parameter is 20. At present, this one parameter is used to set the same number of elements for each CS. If there should be a need to simulate different numbers of elements for different CSs, this could be approached via the definitions of the stimuli. Consider, for instance, the simulation of a discrimination problem involving two CSs. If the first CS should activate twice as many elements as the second, the first stimulus would need to be defined in terms of two letters (i.e., as consisting of two elementary CSs) in the trial definition file (see below). Thus, one way to set up the simulation with the desired number of elements for the two CSs would be to define the first CS as AX and the second as B. A more principled way of defining different numbers of elements for different CSs will be provided in a future version of HMS.

Connection density. One assumption of Harris's (2006) theory is that the elements involved are only partially connected (i.e., there exist certain pairs of elements with no connection to each other). By changing the "Connection density" parameter, one can control the number of connections existing between pairs of elements. Valid entries for this parameter are all real numbers from the closed interval $[0,1]$, with 0 resulting in no connections at all and 1 in complete connection between all elements. Again keeping with the values used by Harris, this parameter defaults to 0.5 .

Attention buffer gain. As already mentioned, the saliencies of the elements inside the attention buffer are increased by boosting their activation values. The "gain" parameter allows specifying the amount of boost resulting from the buffer. More precisely, the increase of activation of each element inside the buffer will be multiplied by the gain parameter value. The default employed is the same used by Harris (2006) — namely, 2.

Beta. This parameter allows for setting the learning rate in the modified learning rule (see Equation 2 on p. 595 of Harris, 2006). The default value for the learning rate is 0.005 .

Advanced settings. Four more parameters can also be employed to change the behavior of the model. Since vary- 
ing these parameters amounts to altering the conceptual/ theoretical flavor of the model, they are only available through the Advanced Settings dialog box (see Figure 1C), which can be invoked by pressing the "Change Advanced Settings" button on the GUI. The parameters, from top to bottom, are described in more detail below.

Attention buffer capacity. There are essentially two ways of specifying the capacity of the attention buffer. One option is to have the simulator set up the capacity on the basis of the activation values of the elements activated by the CS. More precisely, the capacity is set to the sum of the activations of all elements of one particular CS, $S$, such that the sum of the activations of $S$ 's elements is equal to or higher than the sum of the activations of the elements of each of the other CSs. Conceptually, this amounts to the assumption that every single CS employed in the learning situation will always enter the attention buffer completely when presented alone (i.e., that each single CS can be fully attended). This is the automatic option, which is the default for this parameter. As an alternative, the buffer capacity can be set by hand to any real value greater than 0 .

Associations between CSs. In principle, Harris's (2006) theory assumes that the formation of associative connections is not restricted to those between CS and US elements, but that connections can also occur between CS elements. However, according to Harris (personal communication, September 27, 2006), the means by which the learning and impact of CS-CS associations can be implemented formally or computationally have not yet been developed fully, because this would require that time (on the subtrial scale) be comprehensively operationalized, which is beyond the scope of Harris's current version of the model. As a result, it is not currently possible to devise a general algorithm that realizes CS-CS associative learning for the Harris model, and therefore we have refrained from trying to provide such an algorithm. As soon as CS-CS learning is fully developed, we will integrate this option in a future version of HMS. At present, this parameter is just a placeholder and should be left at its default value, "no."

Fraction of common elements. This parameter (for further details, see Harris, 2006, pp. 597 and 599) allows for manipulating the overlap of elements representing different single CSs. The possible values range from 0 to 1 , where 0 indicates no overlap of CS elements (i.e., each single CS activates only its own elements, and the elements of a particular single CS are activated only by that $\mathrm{CS}$ ), and 1 indicates complete overlap of CS elements (i.e., each single CS activates the same elements as every other single CS). The default value for this parameter is 0 . As with the number of elements, currently just one parameter determines the fraction of common elements for all pairs of CSs. A simulation of different fractions of elemental overlap can be approached via appropriate definitions of the stimuli. For instance, if a CS A and a CS B should share two thirds of their elements, but both A and B should share only one third of their elements with a third CS C, the CSs A, B, and C would have to be defined as AXY, BXY, and CX, respectively. The compounds of the different elementary CSs could then be specified by listing all of the letters involved in a compounded CS, without duplicates (i.e., the compound of $\mathrm{A}, \mathrm{B}$, and $\mathrm{C}$ would be written as $\mathrm{ABCXY}$ ). Note that for this approach to yield the desired results, one has to set the fraction of common elements to 0 . Future versions of HMS are planned that would allow for specifying different fractions of common elements for different pairs of CSs more directly.

Simplified learning. Since the way in which US and CS elements compete for entry into the attention buffer is not specified in Harris's (2006) conception of the model, using cutoff values in the learning equations (see Harris, 2006, p. 595, Equation 2) is somewhat arbitrary. As a result, it would also be in accord with the theory to use a simple delta rule (assuming an activation of 1 for all US elements) for learning CS-US associations, and thus to neglect buffer effects (see the Appendix). This parameter allows for specifying whether the simulation should employ the simple delta rule or allow more complex learning by using cutoffs. By default, simple learning is disabled.

The default values for the different parameters are stored in the file default.conf, which is read every time HMS is started and needs to be placed in the same directory as all of the other program files. Storing the default values in an extra file allows for researchers to easily adjust the defaults. For example, if one usually prefers to employ 50 runs instead of 20, it is not necessary to change the parameter value every time when beginning to run the simulator; instead, the corresponding value can be altered in default.conf, and the new value will automatically be loaded every time HMS starts.

Buttons. After having specified all relevant file locations and parameter values, the simulation can be started and controlled by the three buttons at the bottom of the GUI. The functions of the control buttons are as follows.

Initialize. Pressing this button will check whether all necessary information has been specified. If this is the case, all relevant files and fields will be opened and read out. Importantly, the stimuli to be used for training and the evaluation will be read from the trial and evaluation definition files, respectively, and stored in memory. Initialization is a prerequisite to further simulations.

Train. After the simulation has been initialized, learning can take place. Learning proceeds one run at a time. In each run, first the CS (with compounds) and US representations are set up for all CSs (compounds) and the US specified in the definition files. Subsequently, for each trial, all CSs (and compounds) defined for the current phase will be used sequentially to train the model. When all trials for one phase have been processed, the procedure is repeated for the next phase. After the last phase has been executed, learning stops. Procedural and mathematical details regarding the realization of the learning processes used in HMS are given in the Appendix. Once started, learning cannot be interrupted, but will proceed until all iterations (i.e., all runs, phases, and trials) have been processed. Once training has finished, a dialog box will appear informing the user whether the training was successful or whether an error was encountered. Intermediate results for both responses and weights will, however, be registered, and can later be inspected via the evaluation function. 


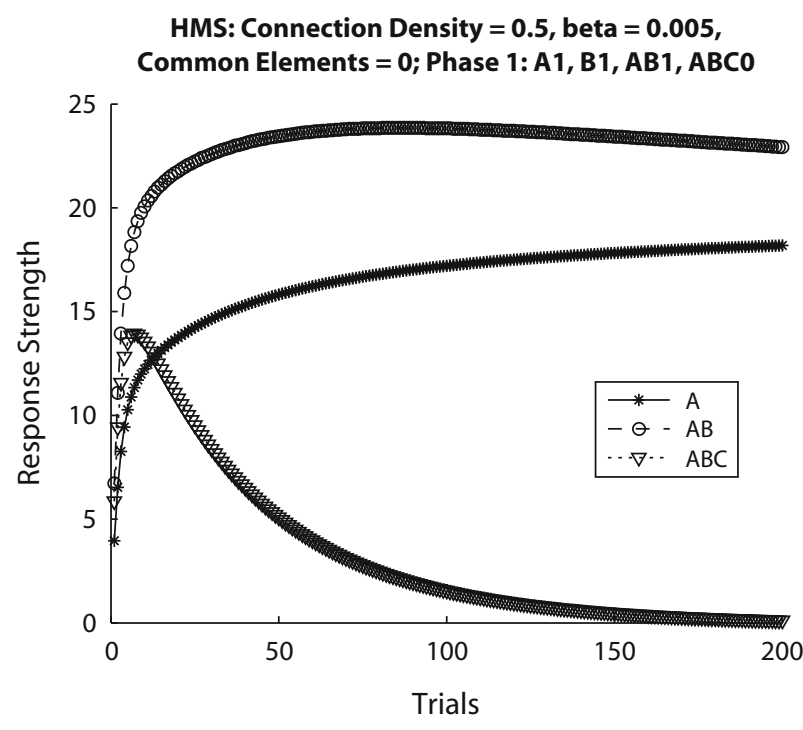

Figure 2. The course of the simulated responses for the discrimination problem $\mathrm{A}+, \mathrm{B}+, \mathrm{AB}+, \mathrm{ABC}-$. Only the responses for $\mathrm{A}$, $A B$, and $A B C$ are shown.

Evaluate. Evaluation basically comprises the graphical display of the course of simulated responses registered during learning. The corresponding plot (see Figure 2 for an example) displays the course of the strength of responding to each of the CS configurations specified in the evaluation definition file. Thus, by appropriately setting up this file, any arbitrary set of CSs (and compounds) can be displayed in the plot. Different curve signatures (i.e., line styles and types of marker) are automatically created for different CS configurations. The legend inside the figure helps identify which evaluation configuration each curve belongs to: The letter beside each different curve indicates the name of the CS configuration represented by that curve. Currently, four different curve styles can be distinguished. To further ease interpretation of the plotted results, a second legend (shown directly above the plot) indicates whether a CS (or compound) was reinforced during training or not. If a CS was paired with the US during training, it is shown with its name trailed by a single 1; if a CS configuration was not paired with the US, it is shown with its name trailed by a single 0 . To facilitate special analyses, the registered response courses are not only graphically displayed, but also written to a comma-separated ASCII text file created in the current directory. The name of the file is automatically generated as simulatedResults $X X X$.txt, where $X X X$ stands for a number uniquely identifying each file, to avoid overwriting previous simulation results with the current ones.

\section{Files}

HMS draws on three types of files to enable the simulation: the trial definition file, the evaluation definition file, and the file specifying the default parameter values. Since the simulation will only work properly if the files are structured correctly, in this section we briefly describe the correct format for each of the different file types.
The trial definition file specifies (1) the names of the CSs used in the simulation and (2) which of these CSs are presented, as well as whether each is presented with the US in a specific trial. The file consists of two sections, the $C S$ section and the trial section. The first of these is preceded by the tag [CS]. Each CS is defined by two lines: The first indicates the name and the second the salience of the particular CS. More precisely, the first substring containing no white spaces (i.e., no space, tab, etc.) in the first line is taken to be the name of an elementary CS. The second line should contain a single floating-point number specifying the salience of the corresponding CS. The higher the salience, the faster and more strongly the associations from the CS elements tend to develop. The CS section is completed by the tag [end-CS]. Note that all CSs used in the simulation have to be defined here. The trial section starts with the tag [trials] and specifies the different trials to be presented during learning. Within the trial section, one or more training phases can be defined. Each training phase begins with the tag [phase] and ends with [end-phase]. In the first line of each phase section, an integer indicates how often this phase shall be executed during the simulation. If this line is missing (i.e., the first line does not contain an integer), the phase will be trained as often as specified in the GUI parameter "Default number of trials per phase." In the remainder of the phase section, two lines define each trial. The first line indicates which CSs are presented on that trial. Each CS is labeled by its name, as defined in the CS section, and separated by a space from other CS names. The second line determines whether or not a US is presented as well: A 0 indicates that during learning the CS configuration defined by the preceding line is not paired with the US, and a 1 indicates that it is. If only one phase is used for the simulation, the [phase] and [end-phase] tags are optional. The trial section is terminated by the tag [endtrials]. Apart from these two sections, the trial definition file may contain any number of lines of comments, which must each begin with “\#” (see Figure 3 for sample trial definition files, with comments).

The evaluation definition file specifies which CSs are to be tested when evaluating the model. Each line specifies a CS configuration, the associative strength of which is determined in the evaluation phase. As in the trial section of the trial definition file, the CSs are named by the substrings in the CS section of the trial definition file and separated by spaces. Importantly, the CS configurations listed in the evaluation definition file are completely independent of those in the trial section of the trial definition file: The evaluation definition file may contain more or fewer configurations than the trial section, and they may be identical to or different from those given in that section. However, all CSs employed in the evaluation definition file must be defined in the CS section of the trial definition file. Just as in the trial definition file, comments may be interspersed as desired in the evaluation definition file (see Figure 4A for a sample evaluation definition file, with comments).

In addition to the files specifying the stimuli used in training and evaluation, HMS requires a file containing default values for the model parameters. This file must be 
A

\# Lines preceded by a hash \# will be ignored \# when evaluating the file for initializing the \# model.

\#

\# The file consists of two sections. In the first \# the elementary CS are defined by giving \# their names (e.g., A) This section is preceded \# by the tag [CS].The second section contains \# the description of the elementary CS present \# on the different trials. This section is \# preceded by the tag [trials]

\# In the CS section every line represents the \# name of ONE elementary CS. More precisely \# the first substring in each line containing no \# white spaces (space, tab, ...) is taken to be the \# name of the elementary CS. The second line \# contains a salience parameter for the CS. [CS]

A

1

B

1

C

[end-CS]

\# The second section specifies how often each \# phase is presented, which CS are presented on \# which trials in which phase and whether they \# are reinforced or not. In the first line of each \# phase section, a integer indicates how often this \# phase shall be executed during simulation. In \# the remaing section, each two lines represent \# one trial. The first line indicates which CS are \# present on that trial. The second line indicates \# whether US is present as well. [trials] A

1

B

1

A B

1

A B C

0

[end-trials]

\section{B}

\# Lines preceded by a hash \# will be ignored \# when evaluating the file for initializing the \# model.

$\#$

\# The file consists of two sections. In the first \# the elementary CS are defined by giving \# their names (e.g., A) This section is preceded \# by the tag [CS].The second section contains \# the description of the elementary CS present \# on the different trials. This section is \# preceded by the tag [trials]

\# In the CS section every line represents the \# name of ONE elementary CS. More precisely \# the first substring in each line containing no \# white spaces (space, tab, ...) is taken to be the \# name of the elementary CS. The second line \# contains a salience parameter for the CS. [CS]

A

1

B

1

C

[end-CS]

\# The second section specifies how often each \# phase is presented, which CS are presented on \# which trials in which phase and whether they \# are reinforced or not. In the first line of each \# phase section, a integer indicates how often this \# phase shall be executed during simulation. In \# the remaing section, each two lines represent \# one trial. The first line indicates which CS are \# present on that trial. The second line indicates \# whether US is present as well.

[trials]

[phase]

400

A

1

B

1

A B

1

A B C

0

[end-phase]

[end-trials]

Figure 3. Examples of trial definition files. (A) File without phases and phase-specific numbers of trials. (B) File employing phases and phase-specific numbers of trials. See the text for details.

named default.conf and needs to be in the same directory as the main program files. Basically, the file consists of two lines that indicate the default values for the general and advanced parameters, respectively. The line holding the values for the general settings must contain six numbers, all separated by at least one white space (space, tab, etc.). Those numbers specify the default parameter values for the general parameters, in the same order those parameters are listed in the GUI, from top to bottom. The line hold- ing the default values for the advanced settings comprises four values that must be separated by at least one white space. The first and the third values should be the numbers for the attention buffer capacity and fraction of common elements, respectively. A negative value for the attention buffer capacity indicates the use of automatic determination of the buffer capacity. The second and fourth values can be set to either "yes" or "no" and specify whether or not associations between CSs and simplified learning (re- 
A

\# Lines preceded by a hash \# will be ignored \# when evaluating the file for initializing the \# model.

\# This file specifies which CS are to be tested \# when evaluating the model. Each of the \# following lines specify a stimulus (which \# might be an elementary or compound \# stimulus) the associative strength of which \# is determined in the evaluation phase.

$A B$

$A B C$
B

\#\# Default configuration file for the AT model simulator. \#\# Do not alter the general structure of the file, but only \#\# the numbers representing the parameter values. \#\# Last changed: 2006/08/12 \#\#

\#\# The first row holds the parameter values for the general settings. \#\# number of runs \# number of trials per run \# elements per CS \# connection density \# attention buffer gain \# beta

$\begin{array}{llllll}20 & 200 & 20 & 0.5 & 2 & 0.005\end{array}$

\#\# The second row holds the the parameter values for the advanced \#\# settings.

\#\# attention buffer capacity: positive numbers map to capacity, \#\# non-positive numbers map to 'automatic' \# associations between \#\# CSs: use 'yes' or 'no' \# fraction of common elements \# simplified \#\# learning: use 'yes' or 'no'

$\begin{array}{llll}-1 & \text { no no }\end{array}$

Figure 4. Examples of (A) an evaluation definition file and (B) default.conf, the file defining the default parameter values. See the text for details.

spectively) will be employed. Besides the lines holding the default values, default.conf may contain comments of the same type as in the two definition files (see Figure 4B for a sample version of default.conf, with comments).

\section{Computational Complexity}

The computationally expensive part of a simulation is the updating of associative weights in each trial. As a consequence, computational complexity will increase with increases in both the number of trials to be simulated and the complexity of the associative updating required. The former factor is controlled by the numbers of runs, phases, and trials: The more runs and trials are requested to be performed, the longer the simulation will take. The complexity of the weight update, on the other hand, is governed by whether simplified or nonsimplified learning is used. The simpler and faster case is weight update with simplified learning. Switching from simplified to nonsimplified learning roughly doubles computational complexity and, thus, execution time. Simulation times range from several seconds to several minutes, depending on the precise combination of the values of the parameters. However, even in complex learning settings with many training trials, simulation times never exceeded $5 \mathrm{~min}$ in the various simulations we conducted with HMS.

\section{Standard Use and an Example}

To give an impression of the overall working of HMS, in this section we provide a brief walk-through of the steps required to simulate a particular discrimination problemfor example, $\mathrm{A}+, \mathrm{B}+, \mathrm{AB}+, \mathrm{ABC}-$. The first step is to write the trial definition file (see Figure 3 ) and the evaluation definition file (see Figure 4A). After that, the corresponding file locations, as well as the desired parameter values, need to be specified via the GUI. After this has been done, the simulator is ready to perform the simulation. By now pressing the "Initialize" and, subsequently, the "Train" button, the associative weights are changed. Finally, the results and the course of response changes can be visualized by pressing the "Evaluate" button. The resulting graphical displays, for the example learning setting with default values provided by the default.conf file in Figure 4B, are shown in Figure 2.

\section{CONCLUSION}

A crucial prerequisite for evaluating and comparing competing models of associative learning (e.g., Harris, 2006; Mackintosh, 1975; Pearce, 1987, 1994; Rescorla \& Wagner, 1972; Wagner, 2003; Wagner \& Brandon, 2001) is the possibility of simulating the learning processes described in the different models. Some of these models are rather easily simulated - for instance, an easy-to-use simulator of the replaced-elements model (Schultheis et al., 2008) can be downloaded free of charge from www.staff.uni-marburg .de/ lachnit/Rapid-REM/. For Harris's recently proposed elemental model, however, such a tool has been missing. First evaluations of Harris's model indicate that it might well turn out to be a very powerful theory of associative learning (see, e.g., Lachnit et al., in press). Therefore, we wanted to provide researchers interested in this topic with a powerful tool to use and further evaluate the model.

To this end, we developed an intuitively operated simulator, called HMS, which not only takes care of setting up all necessary stimulus representations, but also allows for control of the simulation and all its parameters via a GUI. For example, the course and results of a simulation can be made graphically available by a single buttonpress. With HMS, opportunities for simulation and assessment of the Harris (2006) model are easily available. Thus, HMS facilitates evaluating and comparing different associative learning theories and helps in gaining a deeper understanding of the processes and representations involved in associative learning.

\section{AUTHOR NOTE}

The research reported in this article was partially supported by a grant from the Deutsche Forschungsgemeinschaft to H.L. (Grant DFG La 
564/10-4). Many thanks to Justin Harris, who was very helpful in resolving obscurities we had concerning some details of his model. Correspondence concerning this article should be addressed to H. Schultheis, Department of Informatics, Universität Bremen, P.O. Box 330440, 28334 Bremen, Germany (e-mail: schulth@informatik.uni-bremen.de or lachnit@staff uni-marburg.de)

\section{REFERENCES}

Estes, W. K. (1973). Memory and conditioning. In F. J. McGuigan \& D. B. Lumsden (Eds.), Contemporary approaches to conditioning and learning (pp. 265-286). Washington, DC: Winston.

Estes, W. K. (1994). Classification and cognition. Oxford: Oxford University Press.

HARris, J. A. (2006). Elemental representations of stimuli in associative learning. Psychological Review, 113, 584-605.

Lachnit, H., Schultheis, H., KöNig, S., Üngör, M., \& Melchers, K. G. (in press). Comparing elemental and configural associative theories in human causal learning: A case for attention. Journal of Experimental Psychology: Animal Behavior Processes.

MacKInTOSH, N. J. (1975). A theory of attention: Variations in the associability of stimuli with reinforcement. Psychological Review, 82, 276-298.

Pearce, J. M. (1987). A model for stimulus generalization in Pavlovian conditioning. Psychological Review, 94, 61-73.

PeArCe, J. M. (1994). Similarity and discrimination: A selective review and a connectionist model. Psychological Review, 101, 587-607.

Pearce, J. M. (2002). Evaluation and development of a connectionist theory of configural learning. Animal Learning \& Behavior, 30, 73-95.

Pearce, J. M., \& Hall, G. (1980). A model for Pavlovian learning: Variations in the effectiveness of conditioned but not of unconditioned stimuli. Psychological Review, 87, 532-552.

Rescorla, R. A., \& Wagner, A. R. (1972). A theory of Pavlovian conditioning: Variations in the effectiveness of reinforcement and nonreinforcement. In A. H. Black \& W. F. Prokasy (Eds.), Classical conditioning II: Current research and theory (pp. 64-99). New York: Appleton-Century-Crofts.

Schultheis, H., Thorwart, A., \& Lachnit, H. (2008). Rapid-REM: A MATLAB simulator of the replaced-elements model. Behavior Research Methods, 40, 435-441.

Shanks, D. R., Lachnit, H., \& Melchers, K. G. (2008). Representational flexibility and the challenge to elemental theories of learning: Response to commentaries. Behavioural Processes, 77, 451-453.

WAGNER, A. R. (1981). SOP: A model of automatic memory processing in animal behavior. In N. E. Spear \& R. R. Miller (Eds.), Information processing in animals: Memory mechanisms (pp. 5-47). Hillsdale, NJ: Erlbaum.

Wagner, A. R. (2003). Context-sensitive elemental theory. Quarterly Journal of Experimental Psychology, 56B, 7-29.

WAGNER, A. R., \& BRANDON, S. E. (2001). A componential theory of Pavlovian conditioning. In R. R. Mowrer \& S. B. Klein (Eds.), Handbook of contemporary learning theories (pp. 23-64). Mahwah, NJ: Erlbaum.

Wagner, A. R., \& Rescorla, R. A. (1972). Inhibition in Pavlovian conditioning: Application of a theory. In R. A. Boakes \& M. S. Halliday (Eds.), Inhibition and learning (pp. 301-340). London: Academic Press.

\section{NOTE}

1. In designing HMS, we had to find a balance between range of application, on the one hand, and convenience and simplicity, on the other. In the present version, we attached more importance to the latter concern, primarily to encourage as many researchers as possible to engage themselves in evaluating the model of Harris (2006). Therefore, if the HMS software has some limitations, these do not necessarily indicate limitations in the mathematical model itself. For example, the six general settings parameters in the software define all of the stimuli in the simulation identically. Most parameters, however, could be manipulated differentially in the trial definition file.

\section{APPENDIX}

Most of the mathematical and formal underpinnings of the simulation are identical to the formalizations described by Harris (2006). However, some aspects of the simulation are not drawn directly from Harris's article. These aspects pertain to the formulas used to compute changes in associative connections between elements, and they are detailed in the following discussion.

The formulas for updating associations between CS and US elements are given in Equations 2 and 3 on p. 595 of Harris (2006). Whereas Equation 3 has been adopted without change, Equation 2 has been slightly modified in HMS. More precisely, we changed the product in the "else" branch of Equation 2 to the following:

$$
-1 \cdot \omega_{x} \cdot \beta_{y} \cdot\left|\Delta \omega_{y}\right| ;
$$

that is, instead of taking the negative of $\Delta \omega_{y}$, we take the absolute value of it and negate the whole product. This prevents the simulation from yielding unreasonable associative strengths whenever $\Delta \omega_{y}$ becomes negativeas, for example, in the problem $\mathrm{A}+, \mathrm{B}+, \mathrm{AB}+, \mathrm{ABC}-$. At the same time, our formula gives exactly the same results as the original formula for all cases in which $\Delta \omega_{y}$ is not less than zero. As a result, our formula seems to remedy a problem of the original formula without compromising its general idea, and thus, we chose to utilize the new formula in HMS

A second aspect worth noting regarding the learning of CS-US associations concerns the simplification of the weight update process. As mentioned in the text, the formulas given in Equation 2 of Harris (2006) are only one way of updating the weights. Since it has not yet been specified how CS and US elements might compete for entry into the buffer, it is equally in line with Harris's current conception of the model to neglect buffer threshold effects and instead use a simple delta rule for learning. In HMS, the simplified learning rule used is

$$
(\text { gain }-1) \cdot \omega_{x} \cdot \beta_{y} \cdot \Delta \omega_{y},
$$

where "gain" is the factor by which the increase of element activation inside the buffer is multiplied (see the relevant section in the text). Roughly speaking, this formula assumes that all US element activation increases occur both inside and outside the buffer, which amounts to neglect of the buffer threshold effect of learning.

(Manuscript received September 7, 2007;

revision accepted for publication December 7, 2007.) 